\title{
Combining des-gamma-carboxyprothrombin and alpha-fetoprotein for hepatocellular carcinoma diagnosing: an update meta-analysis and validation study
}

\author{
Huaping Chen ${ }^{1, *}$, Siyuan Chen ${ }^{1, *}$, Shan Li ${ }^{1, *}$, Zhijian Chen $^{1}$, Xuan Zhu ${ }^{1}$, Meiyu Dai ${ }^{1}$, \\ Lingxi Kong ${ }^{1}$, Xiaodan Lv ${ }^{1}$, Zhili Huang ${ }^{1}$ and Xue Qin ${ }^{1}$ \\ ${ }^{1}$ Department of Clinical Laboratory, First Affiliated Hospital of Guangxi Medical University, Nanning 530021, Guangxi, China \\ *These authors contributed equally to this work and should be considered as co-first authors
}

Correspondence to: Xue Qin, email: qinxue919@126.com

Keywords: des-gamma-carboxyprothrombin, AFP, hepatocellular cancer, diagnosis, meta-analysis

Received: June 22, $2017 \quad$ Accepted: July 30, $2017 \quad$ Published: August 07, 2017

Copyright: Chen et al. This is an open-access article distributed under the terms of the Creative Commons Attribution License 3.0 (CC BY $3.0)$, which permits unrestricted use, distribution, and reproduction in any medium, provided the original author and source are credited.

\section{ABSTRACT}

Controversies about the combination of des-gamma-carboxyprothrombin (DCP) and alpha-fetoprotein (AFP) for hepatocellular carcinoma diagnosing still exist. Hence, we performed this updated meta-analysis to estimate the diagnostic value of DCP , AFP and DCP + AFP in HCC. In addition, we conducted a validation study to analyze the performance of the candidate makers. After a systematic literature review, 27 studies from $\mathbf{2 0}$ articles were identified from four major databases. The pooled sensitivity and specificity were $69 \%$ and $89 \%$, respectively, for DCP; for AFP, they were $65 \%$ and $88 \%$, respectively; and they were $82 \%$ and $85 \%$, respectively, for DCP + AFP. The values of the area under the curve (AUC) for DCP, AFP, DCP + AFP, respectively, were $0.88,0.75$, and 0.90 . The validation study confirmed that the performance of DCP + AFP (sensitivity $=84 \%$, specificity $=86 \%$; AUC $=0.887$ ) was higher than that of DCP (sensitivity $=76 \%$, specificity $=92 \%$; AUC $=0.843$ ) or AFP (sensitivity $=73 \%$, specificity $=92 \% ;$ AUC $=0.837$ ) alone.

\section{INTRODUCTION}

Hepatocellular carcinoma (HCC) is one of the most (70-90\%) frequent types of primary carcinoma of the liver and it is the third dominating cause of cancer-related mortality among men worldwide [1]. The incidence rate of HCC rises in accordance with increased rates of hepatitis $\mathrm{C}$ virus and hepatitis $\mathrm{B}$ virus infection [2]. Aflatoxin exposure, heavy alcohol drinking, nonalcoholic fatty liver disease, and smoking also contribute to the occurrence and progression of HCC [2].

Serum alpha-fetoprotein (AFP) is commonly used to diagnose HCC. However, owing to its low sensitivity and specificity, AFP testing alone is not recommended in diagnostic assessments of HCC [3-10]. Instead, it is used to combine with other serum or plasma tumor markers, which have been shown to have superior diagnostic abilities [3, 6, 11-15].
Des-gamma-carboxyprothrombin (DCP), an abnormal form of the prothrombin protein, is induced by a deficiency of vitamin $\mathrm{K}$ or antagonist-II [16]. The role of DCP as a biomarker of HCC was first reported in a study published 1984 [17], which found that DCP was present in $91 \%$ of HCC patients but not detectable in other benign liver diseases. Subsequently, numerous studies $[6-8,13$, 18-20] demonstrated that a combined analysis of DCP and AFP led to better prediction of HCC although there are some controversies about the diagnostic accuracy of DCP + AFP.

Thus, we performed an update meta-analysis to contrast the diagnostic performance of DCP alone, AFP alone, and DCP + AFP in the detection of HCC. In addition, we performed a validation study of 45 HCC patients, 42 liver cirrhosis patients, 43 patients with hepatitis virus infections, and 44 normal controls to determine the diagnostic efficacy of these candidate markers. 


\section{RESULTS}

\section{Selection and characteristics of the included studies}

As shown in Figure 1, a search of four databases (PubMed, Embase, the Cochrane Library, and the ISI Web of Science) and a manual search identified 951 relevant articles. Of these, 176 were published before 2000, and 231 were duplicates. After sieving the titles and abstracts, 412 articles about reviews, case reports, letters, nonhuman studies, or unrelated to the topic were excluded. The rest of 132 studies were deemed to satisfactory, and they were read and evaluated carefully for a full-text review. Of these, 112 articles were eliminated for the following reasons: did not contain adequate data to construct a $2 \times 2$ table, did not contain sufficient information on the criteria used to diagnose $\mathrm{HCC}$ or analyzed only serum DCP and AFP alone, or they were published in non-English language periodicals. In total, 27 studies from 20 articles $[11,12,14-16,18-32]$ measured up for this meta-analysis and all of them were reported from 2000 to 2016.

This meta-analysis was included $7507 \mathrm{HCC}$ patients and 5399 controls and the features of eligible studies were showed in Supplementary Table 1. All the included patients had undergone separate tests of serum and plasma levels of DCP and AFP as well as the test results of candidate markers (DCP, AFP, and DCP + AFP) had been analyzed by statistics.

The quality of the studies on the basis of the assessment scores of QUADAS [33-35] is summarized in Supplementary Table 2. Four articles met 10 of the 14 QUADAS norms, nine articles met 11 of the 14 QUADAS norms, six articles met 12 of the 14 QUADAS norms, and one article met 13 of the 14 QUADAS norms. According to the results of the QUADAS assessment, all the included studies were great in quality.

\section{Pooled diagnostic performance of DCP, AFP, DCP + AFP in HCC diagnosing}

Figure 2A-2C shows the sensitivity and specificity of the biomarkers: DCP: sensitivity $\left(\mathrm{I}^{2}=89.6 \%, P=0.0000\right)$ and specificity $\left(\mathrm{I}^{2}=92.1 \%, P=0.0000\right)$; AFP: sensitivity $\left(\mathrm{I}^{2}=69.0 \%, P=0.0000\right)$ and specificity $\left(\mathrm{I}^{2}=90.0 \%, P=\right.$ $0.0000) ; \mathrm{DCP}+$ AFP: sensitivity $\left(\mathrm{I}^{2}=79.9 \%, P=0.0000\right)$ and specificity $\left(\mathrm{I}^{2}=92.8 \%, P=0.0000\right)$. As all the data pointed to significant heterogeneity in the meta-analysis, a random-effects model was chose. When the data were pooled, the sensitivity and specificity of DCP, AFP, and

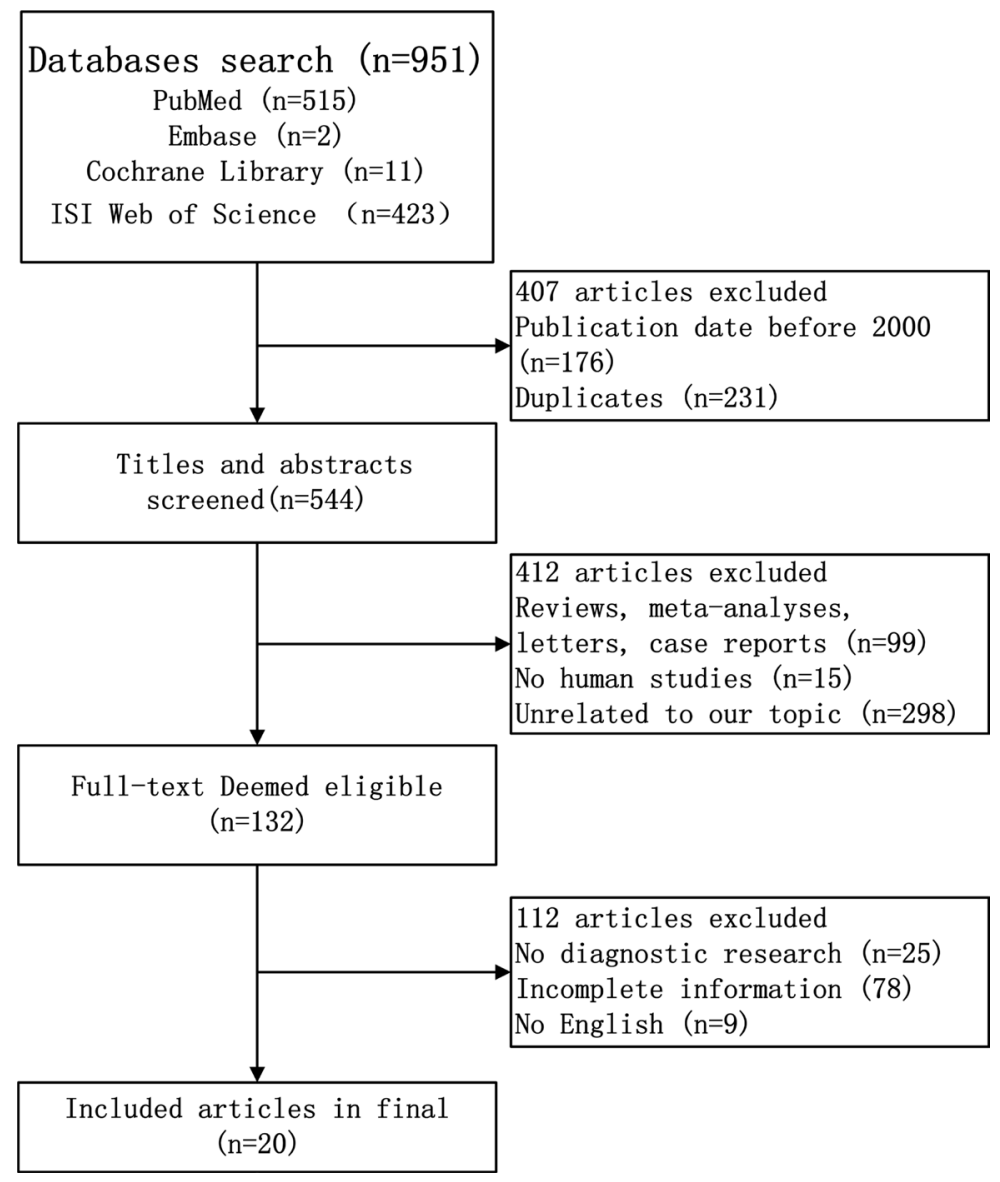

Figure 1: Flow diagram of study selection process for eligible studies. 
DCP + AFP were $0.69(0.68-0.70)$ and $0.89(0.88-0.90)$, $0.65(0.63-0.66)$ and $0.88(0.87-0.89)$, and $0.82(0.81-$ $0.83)$ and $0.85(0.85-0.86)$, respectively.

A
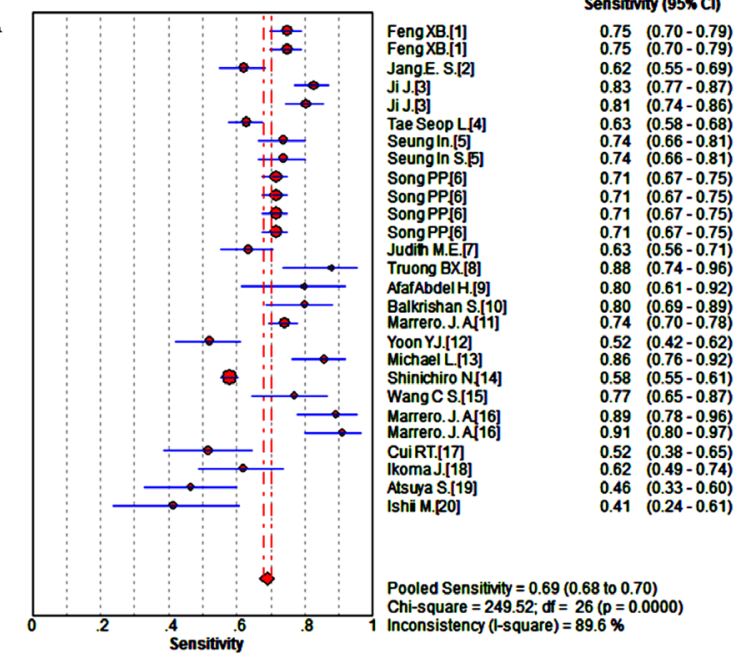

B

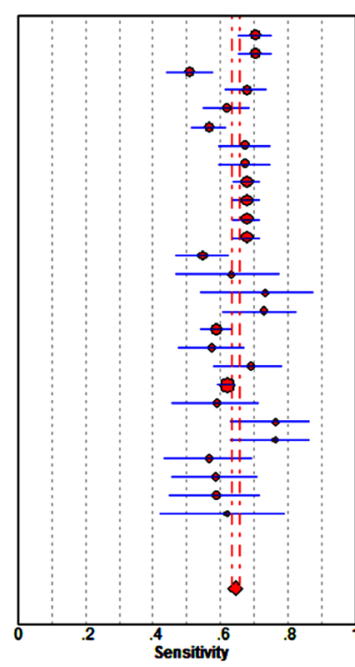

C

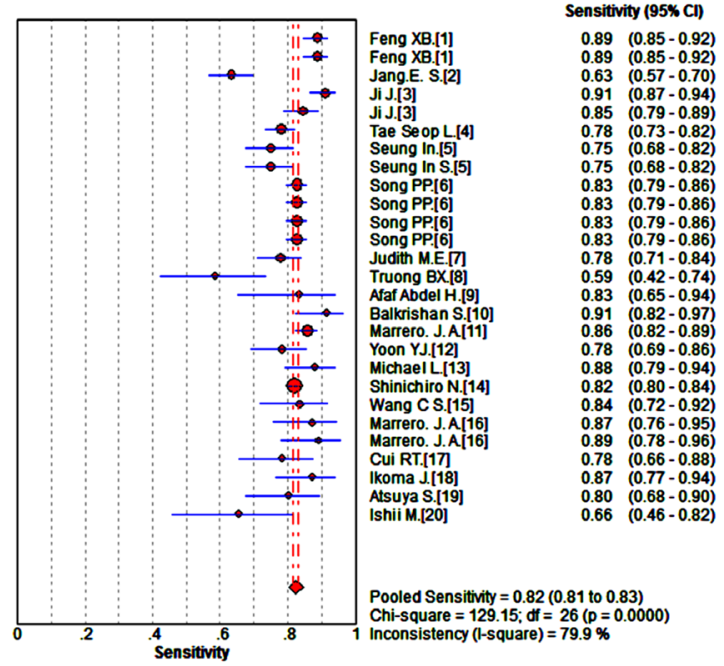

The summary receiver operator characteristic (SROC) curves of DCP, AFP, DCP + AFP are shown in Figures $3 \mathrm{~A}-3 \mathrm{C}$. The results demonstrated that the area
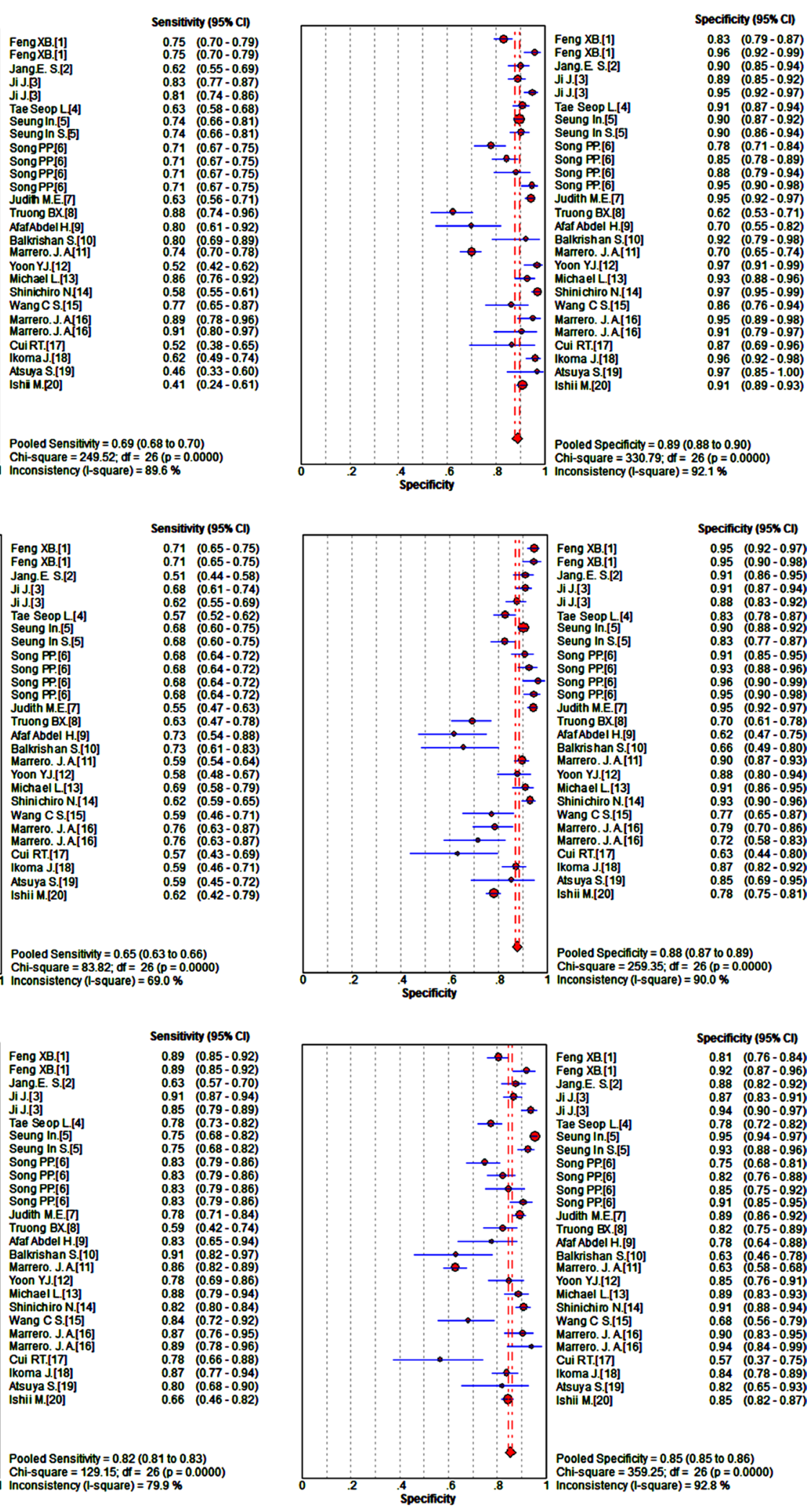

Figure 2: Forest plots of the sensitivity and specificity of candidate makers in the diagnosis of HCC. (A) DCP; (B) AFP; (C) DCP + AFP. 
under the curve (AUC) values for DCP, AFP, DCP + AFP were, respectively, $0.88,0.75$, and 0.90 . The pooled positive likelihood ratios (PLRs), negative likelihood ratios (NLRs), and diagnostic odds ratios (DORs) were 7.28 (5.52-9.61), 0.33 (0.29-0.37), 24.59 (17.98-33.62), respectively, for DCP; 5.07 (3.98-6.45), 0.41 (0.38-0.44), and 12.96 (9.9016.98), respectively, for AFP; and 5.48 (4.31-6.96), 0.22 (0.19-0.25), and 26.45 (19.61-35.68), respectively, for DCP + AFP. The diagnostic performance of the candidate biomarkers are presented in Table 1 . The results suggested that the diagnostic value of DCP + AFP in the detection of $\mathrm{HCC}$ was better than that of either DCP or AFP alone.

\section{Threshold effects and meta-regression analysis of heterogeneity}

To identify potential sources of heterogeneity, tests for threshold effects were conducted by Meta-Disc software. Spearman's correlation coefficient value was $0.295(P=0.135)$ for DCP. It was $-0.007(P=0.971)$ and $0.060(P=0.765)$ for AFP and DCP + AFP, respectively, which suggested there were no threshold effects in this meta-analysis.

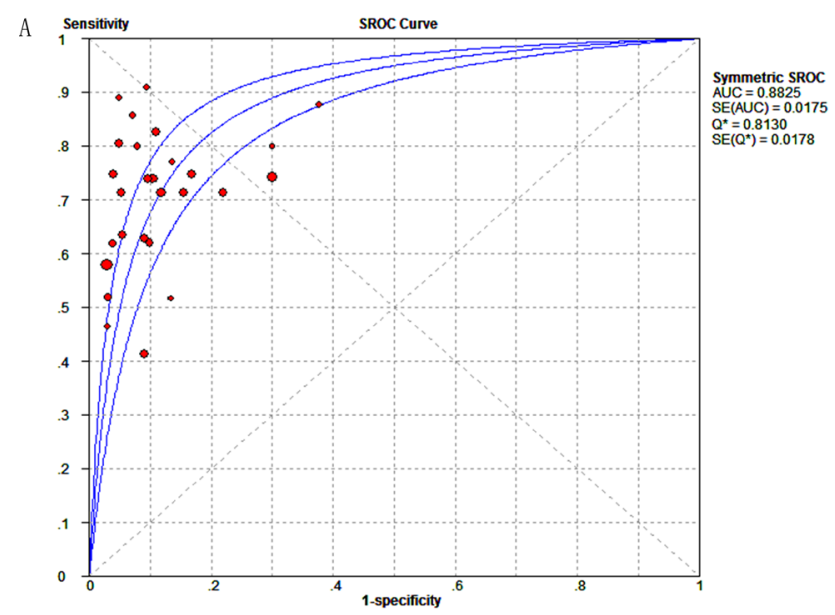

Other than threshold effects, the diversity of study populations in different countries, methodology used, and size of the study population can be potential sources of heterogeneity. To analyze these factors, a meta-regression was performed (Table 2). With regard to DCP, the results revealed no significant heterogeneity in respect of country (coefficient $=-0.132, P=0.1736$ ), methodology (coefficient $=0.044, P=0.7654)$, or size of the study population (coefficient $=0.000, P=0.2863$ ). It proved other factors might lead to the high heterogeneity of DCP. For AFP, they showed no significant heterogeneity in respect of the size of the study population (coefficient $=-0.001, P=0.0525)$. Interestingly, the results of the meta-regression indicated that differences in countries (coefficient $=-0.088, P=0.0356$ ) and methodology (coefficient $=-0.106, P=0.0454$ ) might be the source of heterogeneity in AFP studies.

\section{Sensitivity analysis and publication bias}

Removal of individual studies included in this metaanalysis was evaluated each time to determine the impact of the remaining data set on the sensitivity and specificity.

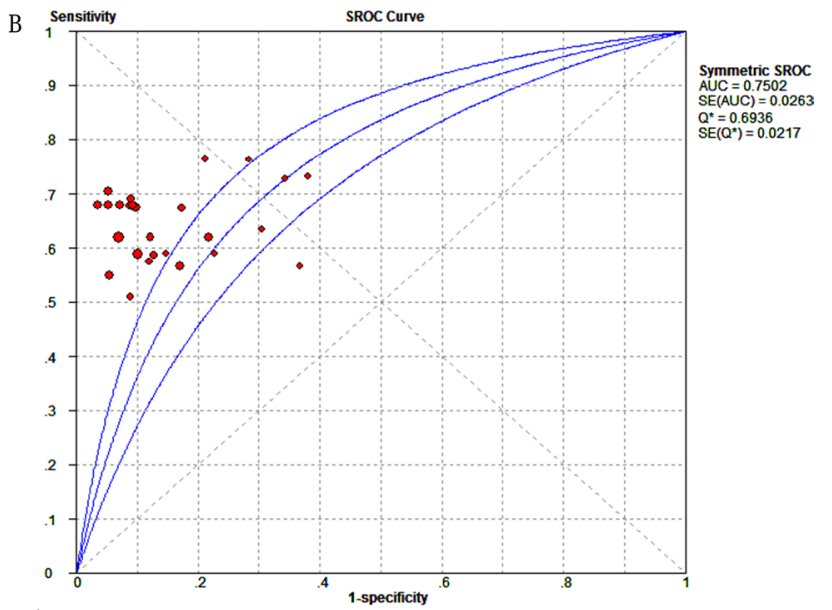

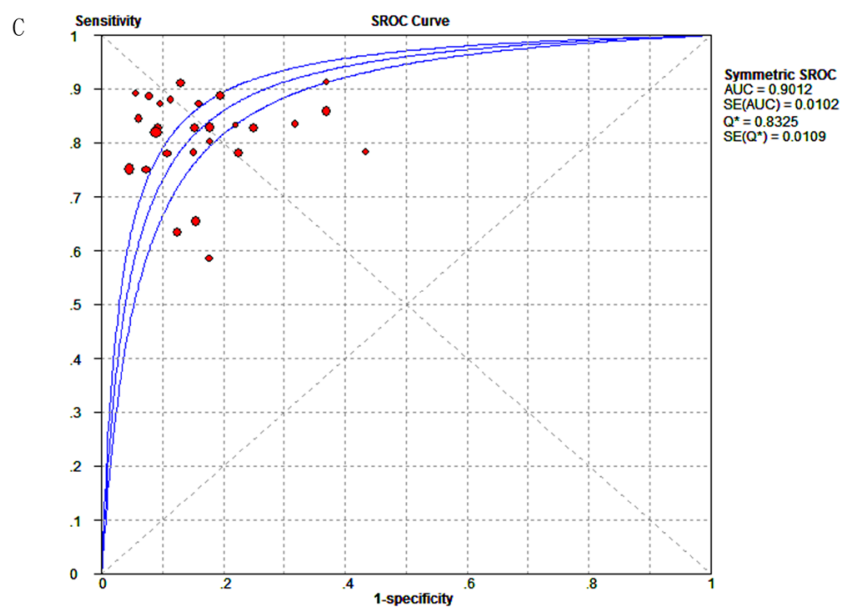

Figure 3: SROC curve of candidate makers for diagnosing HCC. (A) DCP; (B) AFP; (C) DCP + AFP. 
Table 1: Meta-analysis results of DCP, AFP, DCP+AFP

\begin{tabular}{|c|c|c|c|c|c|c|}
\hline Maker & $\begin{array}{c}\mathrm{Se} \\
(95 \% \mathrm{CI}) \\
\mathrm{I}^{2}\end{array}$ & $\begin{array}{c}\text { Sp } \\
(95 \% \mathrm{CI}) \\
\mathbf{I}^{2}\end{array}$ & $\begin{array}{c}\text { PLR } \\
(95 \% \mathrm{CI}) \\
\mathrm{I}^{2}\end{array}$ & $\begin{array}{c}\text { NLR } \\
(95 \% \mathrm{CI}) \\
I^{2}\end{array}$ & $\begin{array}{c}\text { DOR } \\
(95 \% \mathrm{CI}) \\
\mathbf{I}^{2}\end{array}$ & AUC \\
\hline $\mathrm{DCP}$ & $\begin{array}{c}0.69 \\
(0.68-0.70) \\
89.6 \%\end{array}$ & $\begin{array}{c}0.89 \\
(0.88-0.90) \\
92.1 \%\end{array}$ & $\begin{array}{c}7.28 \\
(5.52-9.61) \\
91.6 \%\end{array}$ & $\begin{array}{c}0.33 \\
(0.29-0.37) \\
87.4 \%\end{array}$ & $\begin{array}{c}24.59 \\
(17.98-33.62) \\
84.2 \%\end{array}$ & 0.88 \\
\hline AFP & $\begin{array}{c}0.65 \\
(0.63-0.66) \\
69.0 \%\end{array}$ & $\begin{array}{c}0.88 \\
(0.87-0.90) \\
90.0 \%\end{array}$ & $\begin{array}{c}5.07 \\
(3.98-6.45) \\
89.0 \%\end{array}$ & $\begin{array}{c}0.41 \\
(0.38-0.44) \\
75.2 \%\end{array}$ & $\begin{array}{c}12.96 \\
(9.90-16.98) \\
81.2 \%\end{array}$ & 0.75 \\
\hline $\mathrm{DCP}+\mathrm{AFP}$ & $\begin{array}{c}0.82 \\
(0.81-0.83) \\
79.9 \% \\
\end{array}$ & $\begin{array}{c}0.85 \\
(0.85-0.86) \\
92.8 \%\end{array}$ & $\begin{array}{c}5.48 \\
(4.31-6.96) \\
92.3 \% \\
\end{array}$ & $\begin{array}{c}0.22 \\
(0.19-0.25) \\
81.6 \%\end{array}$ & $\begin{array}{c}26.45 \\
(19.61-35.68) \\
84.9 \%\end{array}$ & 0.90 \\
\hline
\end{tabular}

Se: sensitivity, Sp: specificity, PLR: positive likelihood ratio, NLR: negative likelihood ratio, DOR: diagnostic odds ratio, CI: confidence interval, AUC: area under ROC curve.

The merged results were stable, and they were not altered substantially by individual studies.

As shown in Figure 4A-4C, Begg's funnel plot asymmetry pointed to potential publication bias in the included studies. Thus, a quantitative Egger's linear regression test was conducted to provide statistic evidence of funnel plot symmetry or asymmetry. The results pointed to potential publication biases were detected in $\mathrm{DCP}(P=0.000)$, $\mathrm{AFP}(P=0.000)$ and $\mathrm{DCP}+\mathrm{AFP}(P=0.000)$ studies.
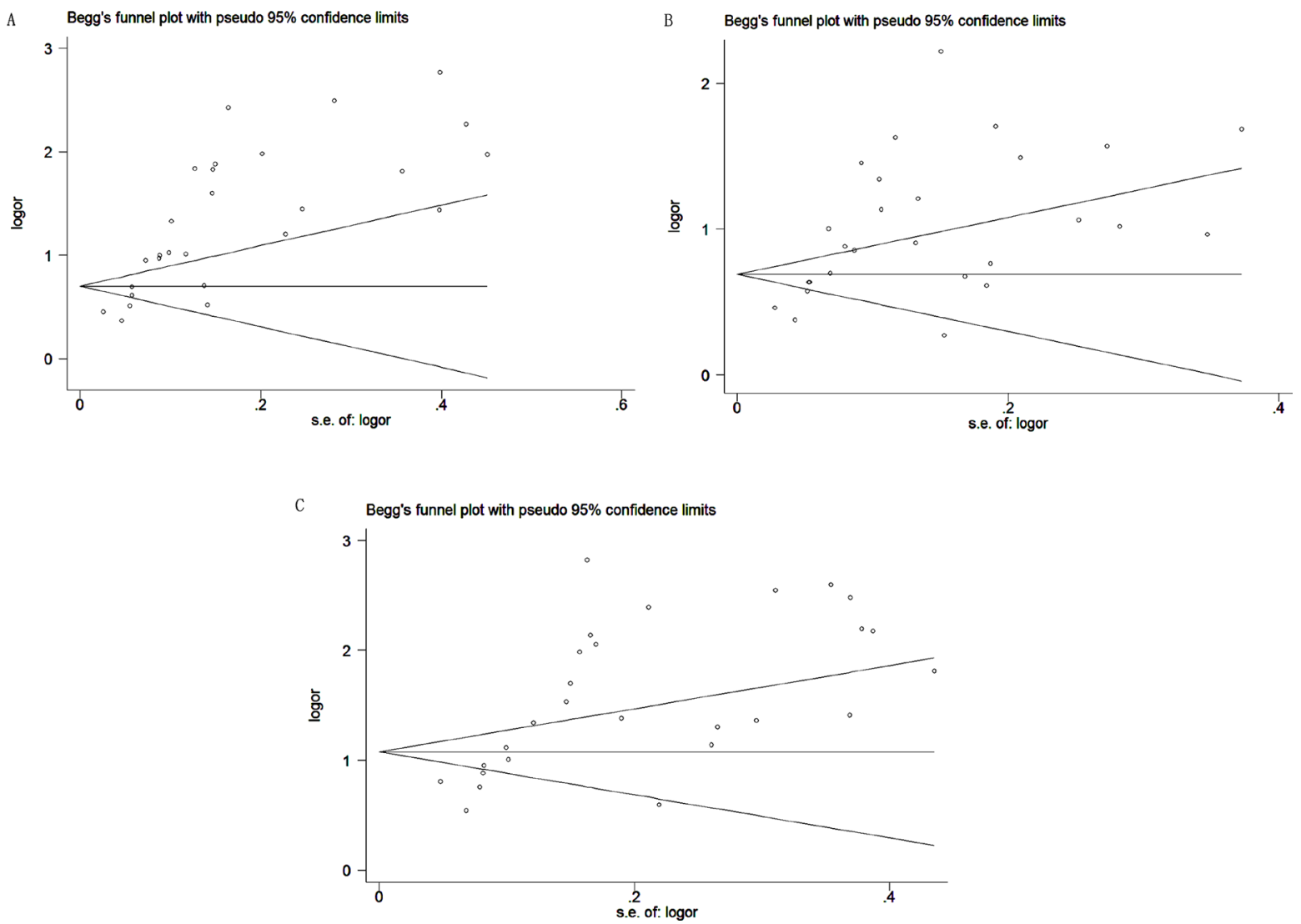

Figure 4: Begg's funnel plot of candidate makers for the studies included in the meta-analysis. (A) DCP; (B) AFP; (C) DCP+AFP. 
Table 2: Meta-regression analyses of the heterogeneity in DCP and AFP

\begin{tabular}{ccccccccccc}
\hline & \multicolumn{3}{c}{ DCP } & \multicolumn{7}{c}{ AFP } \\
\cline { 2 - 11 } Variable & Coeff. & Std.Err. & P-value & RDOR & $\mathbf{( 9 5 \% ) C I}$ & Coeff. & Std.Err. & P-value & RDOR & (95\%)CI \\
\hline Country & -0.132 & 0.0940 & 0.1736 & 0.88 & $(0.72 ; 1.06)$ & -0.088 & 0.0394 & 0.0356 & 0.92 & $(0.84 ; 0.99)$ \\
Method & 0.044 & 0.1454 & 0.7654 & 1.04 & $(0.77 ; 1.41)$ & -0.106 & 0.0454 & 0.0291 & 0.90 & $(0.82 ; 0.99)$ \\
Population & 0.000 & 0.0004 & 0.2863 & 1.00 & $(1.00 ; 1.00)$ & 0.001 & 0.0003 & 0.0525 & 1.00 & $(1.00 ; 1.00)$ \\
\hline
\end{tabular}

Std.Err: standard error, RDOR: ratio of diagnostic odds ratio, CI: confidence interval.

the age of the patients in the different groups: $\mathrm{G} 1$ and $\mathrm{G} 2(P$ $=0.386), \mathrm{G} 1$ and $\mathrm{G} 3(P=0.410), \mathrm{G} 1$ and $\mathrm{G} 4(P=0.709)$, $\mathrm{G} 2$ and $\mathrm{G} 3(P=1.000), \mathrm{G} 2$ and $\mathrm{G} 4(P=0.328), \mathrm{G} 3$ and $\mathrm{G} 4(P=0.289)$. As shown in Figure $5(\mathrm{~A}, \mathrm{~B})$, the serum levels of both DCP and AFP were observably higher in G 1 than in $\mathrm{G} 2(P=0.000$ for both). They were also observably higher in $\mathrm{G} 1$ compared to that in $\mathrm{G} 3(P=0.000$ for both) and meanwhile in $\mathrm{G} 1$ than $\mathrm{G} 4$ ( $P=0.000$ for both). As shown in Supplementary Figure 1, the AUC values of DCP, AFP, and DCP + AFP in distinguishing between HCC (G 1) and liver cirrhosis $(\mathrm{G} 2)$ were 0.860 (95\% CI: $0.770-0.925$, sensitivity [Se]: 0.76 , specificity [Sp]: 0.86$), 0.805(95 \% \mathrm{CI}$ : $0.707-0.882$, Se: 0.73 , Sp: 0.84), and 0.859 (95\% CI: 0.768 0.924 , Se: $0.84, \mathrm{Sp}: 0.77)$, respectively. In distinguishing HCC (G 1) from non-HCC (G 2, 3, and 4), the AUC values of DCP, AFP, and DCP + AFP were 0.843 (95\% CI: 0.7810.894, Se: 0.76, Sp: 0.92), 0.837 (95\% CI: $0.774-0.889$, Se: 0.73 , Sp: 0.92), and 0.887 (95\% CI: 0.830-0.930, Se: 0.84, Sp: 0.86), respectively (Table 3).

\section{DISCUSSION}

The results of this meta-analysis including 27 studies from 20 articles demonstrated that the overall sensitivity and specificity of DCP was, respectively, $69 \%$ and $89 \%$. For AFP, the overall sensitivity and specificity was $65 \%$ and $88 \%$. For DCP + AFP, these values were $82 \%$ and $85 \%$, respectively. Although the specificity of the biomarkers did not differ greatly, the sensitivity of $\mathrm{DCP}+\mathrm{AFP}$ was markedly higher, signifying the superior diagnostic value of DCP + AFP in HCC. DOR is a specific value of PLR and NLR. It is a single indicator of the performance of a trial but independent of prevalence. DOR ranges from zero to infinity, and higher values suggest preferable diagnostic performance. In the present study, the diagnostic odds ratio values of DCP, AFP, and $\mathrm{DCP}+\mathrm{AFP}$ (24.59 vs. 12.96 vs. 26.45) showed that DCP + AFP was better as the indicator of HCC than either DCP or AFP alone. Furthermore, the AUC values of DCP, AFP, and DCP + AFP ( 0.88 vs. 0.75 vs. 0.90$)$ indicated that the accuracy of DCP + AFP in HCC diagnosing was better than that of AFP alone. However, in the findings of the meta-analysis, DCP + AFP were not superior fully to DCP, as shown by the AUC values ( 0.90 vs. 0.88$)$.

Heterogeneity refers to variation in or between studies in systematic reviews. In the present study, the $\mathrm{I}^{2}$ test revealed considerable heterogeneity in DCP, AFP, and DCP + AFP. However, Spearman's correlation coefficient values of DCP, AFP, and DCP + AFP indicated that heterogeneity was caused by no-threshold effects. Thus, we performed a meta-regression, a quantitative method to inspect the impact of variables, such as the size of the study population, to acquire more information about the source of the heterogeneity. The results of the meta-regression showed that differences in test methods and sample size for AFP may contribute to the observed heterogeneity in the application of AFP in HCC detection but not in the application of DCP. The another factor (study population from different countries) analyzed in the meta-regression also could not explain the heterogeneity in the performance of DCP. Therefore, the results of the meta-regression were unable to convincingly explain the heterogeneity in this meta-analysis. In addition, exploration of the potential role of different types of specimens (serum or plasma) or diverse issues of publications of DCP and AFP studies in the metaregression showed that these sources did not contribute to the observed heterogeneity (Supplementary Table 4).

In addition to heterogeneity, publication bias was another limitation in the present study. We executed a sensitivity analysis to determine the stability and reliability of the overall data. Removing each of the 27 studies at a time, the pooled sensitivity varied from 0.68 (0.67 to 0.70$)$ to 0.72 (0.70 to 0.73 ) for DCP, 0.64 (0.63 to 0.65 ) to 0.65 ( 0.63 to 0.66 ) for AFP, and 0.82 ( 0.81 to 0.83 ) to 0.83 (0.82 to 0.84 ) for DCP + AFP. The pooled specificity ranged from 0.88 ( 0.87 to 0.89$)$ to $0.90(0.89$ to 0.91 ) for DCP, 0.87 ( 0.86 to 0.88 ) to 0.89 ( 0.88 to 0.90 ) for AFP, and 0.84 (0.83 to 0.85 ) to 0.87 (0.86 to 0.88 ) for DCP + AFP. Thus, sensitivity analyses in this metaanalysis illuminated that the pooled result and conclusions did not fluctuate by the various decisions that could be made during articles reviewing. It signifies the results of our meta-analysis can be considered with a higher credibility. The results of Egger's linear regression test of DCP, AFP and DCP + AFP in HCC diagnosing were consistent with Begg's funnel plots, which demonstrated that publication bias may be present in this meta-analysis. In the present study, we used an optimal search strategy, which included a comprehensive search of four English databases and a manual search to ensure that no relevant articles were overlooked. However, studies with negative results may remain unpublished, with both authors 
and editors tending to publish positive results of which these two inestimable reasons may lead to publication bias in this article. Therefore, we further performed the tirm and fill method to adjust for publication bias in the present meta-analysis [36]. For DCP in HCC diagnosing studies, the overall analysis merging the theoretical 33 $(27+6)$ studies had little variance with the unredressed 27 studies $($ variance $=0.217, P=0.000$ vs. variance $=$ $0.230, P=0.000)$ and that was similar to DCP + AFP studies $($ variance $=0.297, P=0.000$ vs. variance $=0.341$,
$P=0.000$ ); for AFP researches, none hypothetical study was added and no data were changed between adjusted and unadjusted ones (variance $=0.135, P=0.000$ for both) (Supplementary Figure 2). All of these results implied that the absent articles with negative results did not influence significantly the overall accuracy in our meta-analysis.

In our literature search, there are three similar metaanalyses that also assessed DCP, AFP, and DCP + AFP for $\mathrm{HCC}$ diagnosing [3-5]. Compared with the previous studies [3-5], the advantages of the present meta-analysis

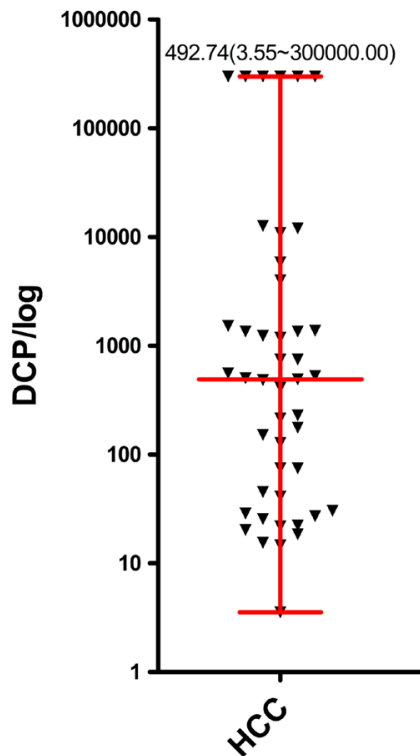

21.24(9.33 1054.23)

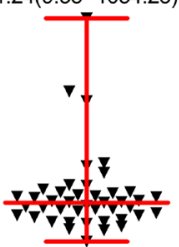

25.69(12.12 158.11)
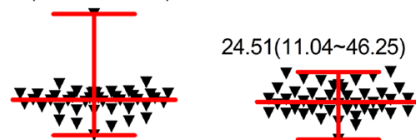

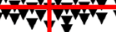

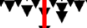

B median of original data (range) $(\mathrm{ng} / \mathrm{ml})$

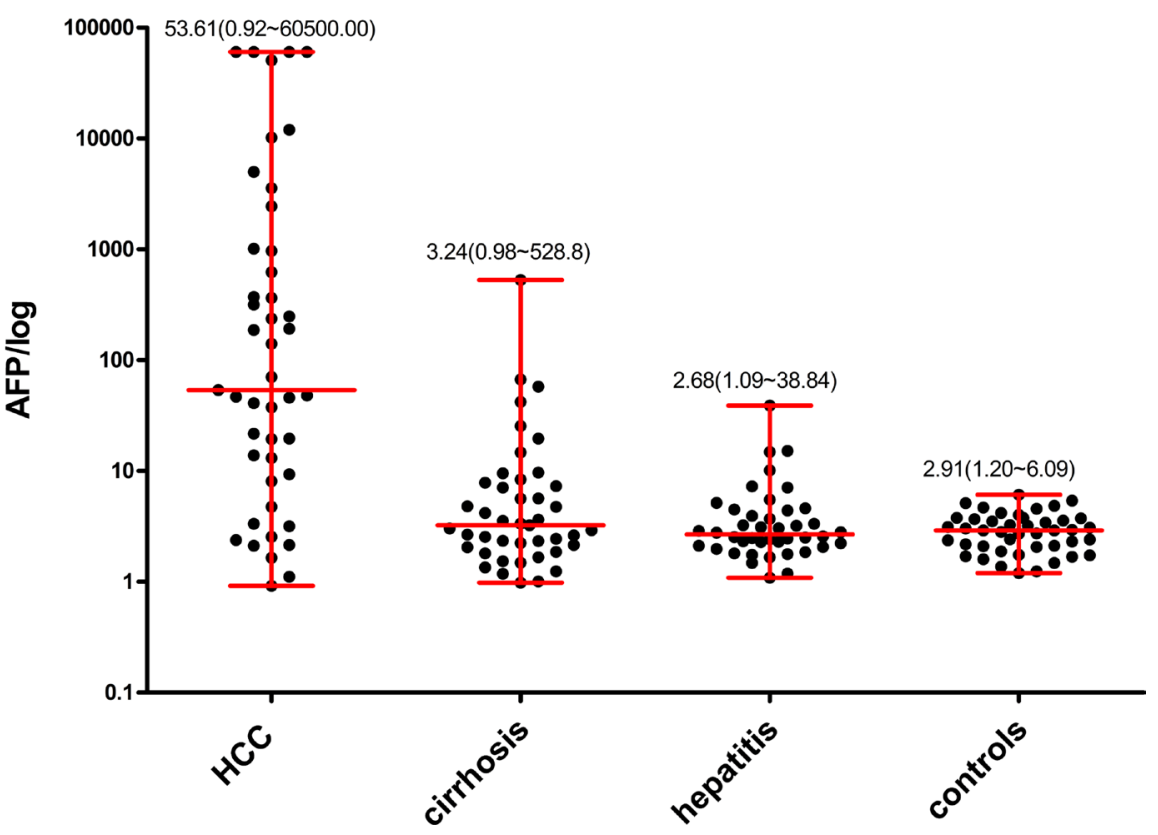

Figure 5: Levels of DCP and AFP in four different groups. DCP was measured in mAU/ml and AFP in ng/ml. Median of original data (minimum to maximum) was shown respectively in the figures. 
Table 3: Diagnostic values of candidate makers for hepatocellular carcinoma

\begin{tabular}{ccccccc}
\hline Makers & AUC & $\mathbf{( 9 5 \%} \mathbf{C I})$ & Cut-off value & Se & Sp & Youden index \\
\hline DCP & & & & & & \\
HCC vs. cirrhosis & 0.860 & $0.770-0.925$ & $40 \mathrm{mAU} / \mathrm{ml}$ & 0.76 & 0.86 & 0.62 \\
HCC vs. no-HCC & 0.843 & $0.781-0.894$ & & 0.76 & 0.92 & 0.67 \\
AFP & & & & & & \\
HCC vs. cirrhosis & 0.805 & $0.707-0.882$ & $11 \mathrm{ng} / \mathrm{ml}$ & 0.73 & 0.84 & 0.57 \\
HCC vs. no-HCC & 0.837 & $0.774-0.889$ & & 0.73 & 0.92 & 0.66 \\
$\quad$ DCP+AFP & & & & & & 0.84 \\
HCC vs. cirrhosis & 0.859 & $0.768-0.924$ & - & 0.77 & 0.61 \\
HCC vs. no-HCC & 0.887 & $0.830-0.930$ & - & 0.84 & 0.86 & 0.70 \\
\hline
\end{tabular}

AUC: area under the curve; CI: confidence interval; Se: sensitivity; Sp: specificity.

are as follows: First, this meta-analysis evaluated the diagnostic performance of DCP + AFP, whereas the previous meta-analyses focused on multiple serum biomarkers for HCC. The present meta-analysis is less ambiguous than previous ones. Second, this meta-analysis included more studies on DCP and AFP and larger samples sizes than previous studies. Third, all the included HCC cases were diagnosed according to gold standard methods to further enhance the validity of the findings. Fourth, the data on DCP and AFP were derived from identical groups to strengthen the comparability of the diagnostic accuracy of the candidate markers (DCP, AFP, and DCP + AFP). Fifth, we included a number of factors (study populations from different countries, test methods, and sample size) not included in previous meta-analyses to explore the source of heterogeneity. Besides, the major orientation in our meta-analysis were similar with a systematic review [37] published in 2014 but different in topics. The present meta-analysis was aimed at the diagnostic performance of DCP + AFP when another aimed to compare the deference between DCP and AFP as biomarkers in diagnosing HCC. In that review [37], the overall evaluations were: sensitivity $=0.63$, specificity $=0.91, \mathrm{AUC}=0.83$ for $\mathrm{DCP}$, sensitivity $=0.59$, specificity $=0.86, \mathrm{AUC}=0.77$ for AFP, sensitivity $=0.81$, specificity $=0.83, \mathrm{AUC}=0.88$ for DCP + AFP. All of the summary results in our metaanalysis were agreed with that in that review. Comparing the systematic review [37], the merits of our meta-analysis were listed below: First, the strict inclusive and exclusive criteria were constituted in this meta-analysis, an Englishlanguage restriction and included HCC patients must be diagnosed by histology, for instance. Second, high quality was performed in each of included articles according to evaluated tool but the precious review was not. Finally, we conducted the analyses of threshold effects and metaregression to explore the heterogeneity.

In our validation study, we evaluated the serum levels of the candidate markers in four different groups (HCC patients, $n=45$; liver cirrhosis patients, $n=43$; patients with the infections of hepatitis virus, $n=42$; and healthy controls, $n=44)$. The results of the validation study demonstrated that "DCP + AFP" was a superior marker to DCP or AFP, which was in accordance with the results of our meta-analysis. The AUC value of DCP + AFP was no significantly higher than that in DCP or AFP ( 0.860 vs. 0.805 vs. 0.859$)$ for distinguishing HCC from liver cirrhosis. As the analysis focus on individuals with no-HCC, the ROC curve and AUC value showed better diagnostic efficiency of DCP + AFP as compared to that of DCP ( 0.887 vs. 0.843$)$ or AFP ( 0.887 vs. 0.837$)$ in distinguishing $\mathrm{HCC}$ from non-HCC. The conclusions of our validation study are also in accordance with those of the majority of studies [11-14, 38-40]. However, the smaller sample number is a limitation of our validation study.

Based on the results of our meta-analysis and validation study, the diagnostic performance of a combination of DCP + AFP is prominent to that of DCP or AFP alone in the detection of HCC. Further research with optimized designs, greater numbers of studies, and larger sample sizes are required to shed light on remaining inconsistencies in variant results of combining desgamma-carboxyprothrombin and alpha-fetoprotein for hepatocellular carcinoma diagnosing.

\section{MATERIALS AND METHODS}

\section{Search strategy}

A systematic literature search was conducted for relevant articles published in PubMed, Excerpta Medica Database (Embase), the Cochrane Library, and ISI Web of Science before 27 December 2016. For AFP, the search terms were AFP and $\alpha$-fetoprotein. For DCP, the search terms were acarboxyprothrombin, PIVKA II, DCP, decarboxyprothrombin, non-carboxylated factor II, protein induced by vitamin $\mathrm{K}$ absence, and antagonists. For HCC, the search terms were liver neoplasms, hepatic neoplasms, hepatocellular cancer, liver cell carcinomas, HCC, small hepatocellular carcinoma, and SHCC. Moreover, the 
references lists of review articles were searched manually to ascertain more relevant studies.

\section{Eligibility criteria and study selection}

To be involved in the meta-analysis, all the articles must fulfill the criteria as follows: (i) contain both individual and combined sensitivity and specificity data on serum or plasma DCP and AFP assays, (ii) consist of patients with a pathologically proven diagnosis of HCC. The exclusion criteria were as follows: (i) reviews, metaanalyses, case reports, or letters; (ii) nonhuman studies; (iii) unrelated to the topic; (iv) incomplete information; and (v) non-English. When study populations overlapped, only the largest sized study or most comprehensive study was incorporated in the concluding analysis.

\section{Data extraction and quality assessment}

The following information was collected from each paper: the name of author, year of publication, country of research, quantity of cases with $\mathrm{HCC}$, quantity of contrasts which may included healthy individuals, patients with hepatitis, cirrhosis or other nonmalignant hepatopathy and non-HCC cancers, assay methods, cut-off points, and the sensitivity and specificity of DCP and AFP alone and their combination (DCP + AFP). The QUADAS (Quality Assessment of Diagnostic Accuracy Studies) tool [3335] was applied to evaluate the quality of the contained studies. Using the 14-item tool, the evaluators rated each item as "Yes", "No," or "Unclear." The reviewers assigned a mark of "1" for "Yes" and "0" for "No" or "Unclear" to each of the 14 items. A high-quality article was deemed one that attained a final Q score of 10 or more.

\section{Validation study of the diagnostic value of DCP, $\mathrm{AFP}$, and DCP + AFP in diagnosing HCC}

The study consisted of HCC patients (G $1, n=45)$, liver cirrhosis patients ( $\mathrm{G} 2, n=43)$, patients with hepatitis virus infections ( $\mathrm{G} \mathrm{3}, n=42$ ), and healthy controls ( $\mathrm{G} 4$, $n=44)$ matched in sex and age from the First Affiliated Hospital of Guangxi Medical University. HCC was diagnosed based on two or more typical imaging modalities or histological examinations. Cirrhosis patients were excluded if they showed evidence of progression to HCC. Patients with hepatitis virus infections were determined by laboratory diagnoses. Healthy subjects who tested negative results of hepatitis B virus surface antigen and with the normal levels of both of aspartate transaminase and alanine transaminase, were included. Individuals with obstructive jaundice and those taking warfarin, as well as samples with hemolysis and lipemia, were also excluded.

Serum levels of DCP were determined using a chemiluminescent immunoassay assay, and AFP concentrations were resolved by electrochemiluminescence immunoassay, according to the manufacturer's instructions.

\section{Statistical Analysis}

\section{Meta-analysis}

Heterogeneity was evaluated by the $\mathrm{I}^{2}$ test. A value of $P<0.1$ and $\mathrm{I}^{2}>50 \%$ were considered indicative of significant heterogeneity. A random effects model was selected in cases of obvious heterogeneity $\left(P<0.1, \mathrm{I}^{2}>\right.$ $50 \%$ ). Otherwise (i.e., in cases of $P \geq 0.1, \mathrm{I}^{2} \leq 50 \%$ ), a fixed effects model was applied. To evaluate the performance of the diagnostic studies, Spearman's correlation coefficient was applied to test for threshold effects and the overall sensitivity, specificity, likelihood ratios, and diagnostic odds ratios (DORs), together with their $95 \%$ confidence intervals (CIs), in addition to their summary receiver operating characteristic (SROCs) curves and area under the curve (AUCs) were calculated by the quantities of true positive, false positive, false negative, and true negative. Furthermore, a meta-regression analysis, sensitivity analysis, and analyses for publication bias were executed simultaneously. All the statistic analyses were conducted utilizing Meta-Disc software (version 1.4) and Stata (version 12.0).

\section{Validation study}

In the validation study, the statistical data analyses were performed with IBM SPSS (version 20), and sectional graphics were executed using GraphPad Prism (version 5.01). A chi-square test was applied to compare classified variables. The Mann-Whitney test was performed to compare differences between two groups. A two-tailed test of $P$-value $<0.05$ was considered as statistical differences. ROC curve and binary logistic regression analyses were used to calculate the areas under the ROC (AUROC) of DCP + AFP. The AUC was served for comparing the performance of DCP, AFP, and DCP + AFP. The cutoff values for DCP and AFP were 40mAU/ $\mathrm{ml}$ and $11 \mathrm{ng} / \mathrm{ml}$, in accordance with the manufacturer's instructions. As hepatic cirrhosis is known to be a pathogenic factor that is closely related to $\mathrm{HCC}$, in this study, we analyzed the AUC, sensitivity, specificity, and Youden index in patients with and without HCC (G 1 vs. G 2, 3, and 4) and in HCC patients and liver cirrhosis patients ( $\mathrm{G} 1$ vs. $\mathrm{G} 2$ ).

\section{Abbreviations}

DCP: des-gamma-carboxyprothrombin; AFP: alpha-fetoprotein; HCC: Hepatocellular carcinoma; AUC: area under the curve; QUADAS: Quality Assessment of Diagnostic Accuracy Studies; Se: sensitivity; Sp: specificity; PLRs: positive likelihood ratios; NLRs: negative likelihood ratios; DORs: diagnostic odds ratios; CIs: confidence intervals. 


\section{Author contributions}

$\mathrm{XQ}$ and SL conceived and designed the metaanalysis and validation study. $\mathrm{HC}, \mathrm{SC}$ and $\mathrm{ZC}$ performed the literature search and quality assessment. LK and XL implemented the data extraction. $\mathrm{ZH}$ and $\mathrm{ZC}$ executed the validation study. MD, HC conducted the statistical analyses. $\mathrm{HC}$ and $\mathrm{SC}$ wrote the manuscript.

\section{ACKNOWLEDGMENTS}

We thank the authors of the primary articles included in our meta-analysis. We could not perform the review without their previous studies. This work is subsidized by National Natural Science Foundation of China (NO.81460431) and Guangxi natural science funds (2013GXNSFAA253001).

\section{CONFLICTS OF INTEREST}

The authors declare no conflicts of interest.

\section{FUNDING}

This work is supported by National Natural Science Foundation of China (NO.81460431) and Guangxi natural science funds (2013GXNSFAA253001).

\section{REFERENCES}

1. Torre LA, Bray F, Siegel RL, Ferlay J, Lortet-Tieulent J, Jemal A. Global cancer statistics, 2012. CA Cancer J Clin. 2015; 65:87-108. https://doi.org/10.3322/caac.21262.

2. El-Serag HB. Hepatocellular carcinoma. N Engl J Med. 2011; 365:1118-27. https://doi.org/10.1056/ NEJMra1001683.

3. Hussein TD. Serological tumor markers of hepatocellular carcinoma: a meta-analysis. Int J Biol Markers. 2015; 30:e32-42. https://doi.org/10.5301/jbm.5000119.

4. Huang TS, Shyu YC, Turner R, Chen HY, Chen PJ. Diagnostic performance of alpha-fetoprotein, lens culinaris agglutinin-reactive alpha-fetoprotein, des-gamma carboxyprothrombin, and glypican-3 for the detection of hepatocellular carcinoma: a systematic review and meta-analysis protocol. Syst Rev. 2013; 2:37. https:/doi. org/10.1186/2046-4053-2-37.

5. Hu B, Tian X, Sun J, Meng X. Evaluation of individual and combined applications of serum biomarkers for diagnosis of hepatocellular carcinoma: a meta-analysis. Int J Mol Sci. 2013; 14:23559-80. https://doi.org/10.3390/ ijms141223559.

6. Viggiani V, Palombi S, Gennarini G, D'Ettorre G, De Vito C, Angeloni A, Frati L, Anastasi E. Protein induced by vitamin $\mathrm{K}$ absence or antagonist-II (PIVKA-II) specifically increased in Italian hepatocellular carcinoma patients. Scand J Gastroenterol. 2016; 51:1257-62. https://doi.org/10.1080/00365521.2016.1183705.

7. Sultanik P, Ginguay A, Vandame J, Popovici T, Meritet JF, Cynober L, Pol S, Bories PN. Diagnostic accuracy of desgamma-carboxy prothrombin for hepatocellular carcinoma in a French cohort using the Lumipulse(R) G600 analyzer. J Viral Hepat. 2016. https://doi.org/10.1111/jvh.12622.

8. Pote N, Cauchy F, Albuquerque M, Voitot H, Belghiti J, Castera L, Puy H, Bedossa P, Paradis V. Performance of PIVKA-II for early hepatocellular carcinoma diagnosis and prediction of microvascular invasion. J Hepatol. 2015; 62:848-54. https://doi.org/10.1016/j.jhep.2014.11.005.

9. Abd El Gawad IA, Mossallam GI, Radwan NH, Elzawahry HM, Elhifnawy NM. Comparing prothrombin induced by vitamin $\mathrm{K}$ absence-II (PIVKA-II) with the oncofetal proteins glypican-3, Alpha feto protein and carcinoembryonic antigen in diagnosing hepatocellular carcinoma among Egyptian patients. J Egypt Natl Canc Inst. 2014; 26:79-85. https://doi.org/10.1016/j.jnci.2014.01.001.

10. Baek YH, Lee JH, Jang JS, Lee SW, Han JY, Jeong JS, Choi JC, Kim HY, Han SY. Diagnostic Role and Correlation with Staging Systems of PIVKA-II compared with AFP. Hepato-Gastroenterology. 2009; 56:763-7.

11. Lim TS, Kim DY, Han KH, Kim HS, Shin SH, Jung KS, Kim BK, Kim SU, Park JY, Ahn SH. Combined use of AFP, PIVKA-II, and AFP-L3 as tumor markers enhances diagnostic accuracy for hepatocellular carcinoma in cirrhotic patients. J Hepatol. 2016; 51:344-53. https://doi.org/10.3109 /00365521.2015.1082190.

12. Jang ES, Jeong SH, Kim JW, Choi YS, Leissner P, Brechot C. Diagnostic Performance of Alpha-Fetoprotein, Protein Induced by Vitamin K Absence, Osteopontin, Dickkopf-1 and Its Combinations for Hepatocellular Carcinoma. PLoS One. 2016; 11:e0151069. https://doi. org/10.1371/journal.pone.0151069.

13. Caviglia GP, Abate ML, Petrini E, Gaia S, Rizzetto M, Smedile A. Highly sensitive alpha-fetoprotein, Lens culinaris agglutinin-reactive fraction of alpha-fetoprotein and desgamma-carboxyprothrombin for hepatocellular carcinoma detection. Hepatology Research. 2016; 46:E130-E5. https://doi.org/10.1111/hepr.12544.

14. Seo SI, Kim HS, Kim WJ, Shin WG, Kim DJ, Kim KH, Jang MK, Lee JH, Kim JS, Kim HY, Kim DJ, Lee MS, Park CK. Diagnostic value of PIVKA-II and alphafetoprotein in hepatitis B virus-associated hepatocellular carcinoma. World J Gastroenterol. 2015; 21:3928-35. https://doi.org/10.3748/wjg.v21.i13.3928.

15. Song $\mathrm{P}$, Feng $\mathrm{X}$, Inagaki $\mathrm{Y}$, Song $\mathrm{T}$, Zhang $\mathrm{K}$, Wang $\mathrm{Z}$, Zheng S, Ma K, Li Q, Kong D, Wu Q, Zhang T, Zhao X, et al. Clinical utility of simultaneous measurement of alpha-fetoprotein and des-gamma-carboxy prothrombin for diagnosis of patients with hepatocellular carcinoma 
in China: A multi-center case-controlled study of 1,153 subjects. Biosci Trends. 2014; 8:266-73.

16. Feng X, Song P, Bie P, Jiang P, Ma K, Li X, Wang S, Wang Z, Tang W, Zheng S. Des-gamma-Carboxyprothrombin Plasma Level in Diagnosis of Hepatocellular Carcinoma in a Chinese Population Undergoing Surgery. Medical Science Monitor. 2016; 22:1663-72. https://doi.org/10.12659/ msm.895483.

17. Liebman HA, Furie BC, Tong MJ, Blanchard RA, Lo KJ, Lee SD, Coleman MS, Furie B. Des-gamma-carboxy (abnormal) prothrombin as a serum marker of primary hepatocellular carcinoma. N Engl J Med. 1984; 310:1427-31. https://doi.org/10.1056/NEJM198405313102204.

18. Ji J, Wang H, Li Y, Zheng L, Yin Y, Zou Z, Zhou F, Zhou W, Shen F, Gao C. Diagnostic Evaluation of DesGamma-Carboxy Prothrombin versus alpha-Fetoprotein for Hepatitis B Virus-Related Hepatocellular Carcinoma in China: A Large-Scale, Multicentre Study. Plos One. 2016; 11. https://doi.org/10.1371/journal.pone.0153227.

19. Ertle JM, Heider D, Wichert M, Keller B, Kueper R, Hilgard P, Gerken G, Schlaak JF. A Combination of alpha-Fetoprotein and Des-gamma-Carboxy Prothrombin Is Superior in Detection of Hepatocellular Carcinoma. Digestion. 2013; 87:121-31. https://doi. org/10.1159/000346080.

20. Marrero JA, Feng Z, Wang Y, Nguyen MH, Befeler AS, Roberts LR, Reddy KR, Harnois D, Llovet JM, Normolle D, Dalhgren J, Chia D, Lok AS, et al. alphaFetoprotein, Des-gamma Carboxyprothrombin, and Lectin-Bound alpha-Fetoprotein in Early Hepatocellular Carcinoma. Gastroenterology. 2009; 137:110-8. https://doi. org/10.1053/j.gastro.2009.04.005.

21. Truong BX, Yano Y, Van VT, Seo Y, Nam NH, Trach NK, Utsumi T, Azuma T, Hayashi Y. Clinical utility of protein induced by vitamin $\mathrm{K}$ absence in patients with chronic hepatitis B virus infection. Biomed Rep. 2013; 1:122-8. https://doi.org/10.3892/br.2012.4.

22. Hady AA, El Shanawany F, Hassan MM, Anas A, Mostafa I, Hadi AA. Evaluation of Human Telomerase Activity as a Novel Tumor Marker for Hepatocellular Carcinoma. Life Science Journal-Acta Zhengzhou University Overseas Edition. 2010; 7:153-61.

23. Sharma B, Srinivasan R, Chawla YK, Kapil S, Saini N, Singla B, Chakraborthy A, Kalra N, Duseja A, Dhiman RK. Clinical utility of prothrombin induced by vitamin $\mathrm{K}$ absence in the detection of hepatocellular carcinoma in Indian population. Hepatol Int. 2010; 4:569-76. https://doi.org/10.1007/s12072-010-9186-2.

24. Yoon YJ, Han KH, Kim DY. Role of serum prothrombin induced by vitamin $\mathrm{K}$ absence or antagonist-II in the early detection of hepatocellular carcinoma in patients with chronic hepatitis B virus infection. Scand J Gastroenterol. 2009; 44:861-6. https://doi. org/10.1080/00365520902903034.
25. Volk ML, Hernandez JC, Su GL, Lok AS, Marrero JA. Risk factors for hepatocellular carcinoma may impair the performance of biomarkers: a comparison of AFP, DCP, and AFP-L3. Cancer Biomark. 2007; 3:79-87.

26. Nakamura S, Nouso K, Sakaguchi K, Ito YM, Ohashi Y, Kobayashi Y, Toshikuni N, Tanaka H, Miyake Y, Matsumoto E, Shiratori Y. Sensitivity and Specificity of Des-GammaCarboxy Prothrombin for Diagnosis of Patients with Hepatocellular Carcinomas Varies According to Tumor Size. Am J Gastroenterol. 2006; 101:2038-43. https://doi. org/10.1111/j.1572-0241.2006.00681.x.

27. Wang CS, Lin CL, Lee HC, Chen KY, Chiang MF, Chen HS, Lin TJ, Liao LY. Usefulness of serum des-gammacarboxy prothrombin in detection of hepatocellular carcinoma. World J Gastroenterol. 2005; 11:6115-9.

28. Marrero JA, Su GL, Wei W, Emick D, Conjeevaram HS, Fontana RJ, Lok AS. Des-gamma carboxyprothrombin can differentiate hepatocellular carcinoma from nonmalignant chronic liver disease in american patients. Hepatology. 2003; 37:1114-21. https://doi.org/10.1053/jhep.2003.50195.

29. Cui R, Wang B, Ding H, Shen H, Li Y, Chen X. Usefulness of determining a protein induced by vitamin $\mathrm{K}$ absence in detection of hepatocellular carcinoma. Chin Med J (Engl). 2002; 115:42-5.

30. Ikoma J, Kaito M, Ishihara T, Nakagawa N, Kamei A, Fujita N, Iwasa M, Tamaki S, Watanabe S, Adachi Y. Early diagnosis of hepatocellular carcinoma using a sensitive assay for serum des-gamma-carboxy prothrombin: a prospective study. Hepatogastroenterology. 2002; 49:235-8.

31. Shimizu A, Shiraki K, Ito T, Sugimoto K, Sakai T, Ohmori S, Murata K, Takase K, Tameda Y, Nakano T. Sequential fluctuation pattern of serum des-gamma-carboxy prothrombin levels detected by high-sensitive electrochemiluminescence system as an early predictive marker for hepatocellular carcinoma in patients with cirrhosis. Int J Mol Med. 2002; 9:245-50.

32. Ishii M, Gama H, Chida N, Ueno Y, Shinzawa H, Takagi T, Toyota T, Takahashi T, Kasukawa R. Simultaneous measurements of serum alpha-fetoprotein and protein induced by vitamin $\mathrm{K}$ absence for detecting hepatocellular carcinoma. South Tohoku District Study Group. Am J Gastroenterol. 2000; 95:1036-40.

33. Mann R, Hewitt CE, Gilbody SM. Assessing the quality of diagnostic studies using psychometric instruments: applying QUADAS. Soc Psychiatry Psychiatr Epidemiol. 2009; 44:300-7. https://doi.org/10.1007/s00127-008-0440-z.

34. Whiting PF, Weswood ME, Rutjes AW, Reitsma JB, Bossuyt PN, Kleijnen J. Evaluation of QUADAS, a tool for the quality assessment of diagnostic accuracy studies. BMC Med Res Methodol. 2006; 6:9. https://doi. org/10.1186/1471-2288-6-9.

35. Whiting P, Rutjes AW, Reitsma JB, Bossuyt PM, Kleijnen J. The development of QUADAS: a tool for the quality assessment of studies of diagnostic accuracy included in 
systematic reviews. BMC Med Res Methodol. 2003; 3:25. https://doi.org/10.1186/1471-2288-3-25.

36. Duval S, Tweedie R. Trim and fill: A simple funnel-plotbased method of testing and adjusting for publication bias in meta-analysis. Biometrics. 2000; 56:455-63.

37. Li C, Zhang Z, Zhang P, Liu J. Diagnostic accuracy of desgamma-carboxy prothrombin versus alpha-fetoprotein for hepatocellular carcinoma: A systematic review. Hepatol Res. 2014; 44:E11-25. https://doi.org/10.1111/hepr.12201.

38. Yu R, Ding S, Tan W, Tan S, Tan Z, Xiang S, Zhou Y, Mao Q, Deng G. Performance of Protein Induced by Vitamin K Absence or Antagonist-II (PIVKA-II) for Hepatocellular Carcinoma Screening in Chinese Population. Hepat Mon. 2015; 15:e28806. https://doi.org/10.5812/ hepatmon.28806v2.
39. Choi JY, Jung SW, Kim HY, Kim M, Kim Y, Kim DG, Oh EJ. Diagnostic value of AFP-L3 and PIVKA-II in hepatocellular carcinoma according to total-AFP. World J Gastroenterol. 2013; 19:339-46. https://doi.org/10.3748/ wjg.v19.i3.339.

40. Beale G, Chattopadhyay D, Gray J, Stewart S, Hudson M, Day C, Trerotoli P, Giannelli G, Manas D, Reeves H. AFP, PIVKAII, GP3, SCCA-1 and follisatin as surveillance biomarkers for hepatocellular cancer in non-alcoholic and alcoholic fatty liver disease. Bmc Cancer. 2008; 8. https:// doi.org/10.1186/1471-2407-8-200. 